\title{
Strates
}

STRATES Matériaux pour la recherche en sciences sociales

15 | 2008

Union européenne - Voisinages. La quête d'une intégration régionale

\section{L'Europe, un continent d'immigration malgré lui}

\section{Catherine Wihtol de Wenden}

\section{(2) OpenEdition \\ Journals}

Édition électronique

URL : http://journals.openedition.org/strates/6530

DOI : $10.4000 /$ strates.6530

ISSN : 1777-5442

Éditeur

Laboratoire Ladyss

Édition imprimée

Date de publication : 1 janvier 2008

Pagination : 59-71

ISSN : 0768-8067

\section{Référence électronique}

Catherine Wihtol de Wenden, «L'Europe, un continent d'immigration malgré lui », Strates [En ligne], 15 | 2008, mis en ligne le 04 mars 2013, consulté le 08 septembre 2020. URL : http://

journals.openedition.org/strates/6530; DOI : https://doi.org/10.4000/strates.6530

Ce document a été généré automatiquement le 8 septembre 2020

Tous droits réservés 


\title{
L'Europe, un continent d'immigration malgré lui
}

\author{
Catherine Wihtol de Wenden
}

1 L'Europe a longtemps été une terre de départ vers le nouveau monde et les colonies avant de devenir, dans le désordre, une terre d'accueil. En devenant l'une des premières destinations pour l'immigration au monde, l'Europe peine à définir ses politiques de flux et les modalités du vivre ensemble, aux prises avec les difficultés de l'Union à mettre en œuvre des instruments de régulation des flux en commun avec les pays proches, avec les influences contradictoires du vieillissement, des pénuries de main-d'œuvre, du contrôle d'une opinion publique gagnée par le syndrome sécuritaire et les défis de l'ethnicisation de la pauvreté. Confrontée à la mondialisation des flux migratoires, l'Europe est un carrefour de liens: familiaux, économiques, géographiques, historiques, culturels divers avec les régions de départ et de transit mais continue souvent à considérer l'immigration comme une donnée temporaire alors qu'elle est devenue constitutive de son identité.

2 Les flux mondialisés qui touchent l'Europe sont le fruit de la conjonction d'une pluralité de facteurs survenus depuis ces vingt dernières années :

3 - le passage, pour les pays d'Europe du Sud et de l'Est, du statut de pays d'émigration à celui de pays d'immigration ou de transit, certains pays étant devenus l'un et l'autre à la fois ;

4 - la généralisation progressive des passeports, à l'exception de rares pays qui les distribuent encore parcimonieusement. Ce phénomène a entraîné une généralisation $\mathrm{du}$ droit de sortie, alors que le droit d'entrer dans les pays riches devenait de plus en plus contrôlé (visas) ;

5 - l'explosion de la demande d'asile dans les années 1990, dans des proportions inconnues jusque-là (Afrique des grands lacs, Balkans, Proche et Moyen-Orient, Amérique caraïbe), atteignant plus de 500000 demandes par an avant de connaître une rapide décrue depuis les années 2000 ; 
6 - l'activation de réseaux transnationaux légaux et illégaux, à l'origine de migrations en chaîne ${ }^{1}$, d'origine diasporique ou résultant d'une économie du passage ;

7 - le développement de migrations pendulaires ${ }^{2}$ d'allers-retours où l'on ne part plus définitivement mais pour de courtes durées avec pour but de rester dans de meilleures conditions chez soi, d'Est en Ouest de l'Europe, notamment car la liberté de circulation le permet ;

8 - la proximité géographique, rendue plus aisée par la baisse généralisée du coût des transports, aériens notamment, par une image de l'Europe véhiculée par les chaînes de télévision et de radio reçues dans les pays de départ, par les marchés locaux approvisionnés en produits manufacturés occidentaux et les transferts de fonds des migrants (14 milliards d'euros envoyés par les immigrés d'Europe vers leurs pays d'origine en 2005). Tout cela suscite une « envie d'Europe » dans les régions d'origine les plus enclavées.

9 Leur profil des migrants s'est beaucoup diversifié au cours de ces dernières années Ceux qui partent sont ceux qui disposent d'un réseau, de famille installée à l'étranger, d'un pécule quand le franchissement des frontières est impossible par les voies légales : s'il n'y a pas de réseau, point de migrations. La seule exception à cette mobilité transnationalisée est la migration forcée des réfugiés. Ce sont moins la pression démographique (d'ailleurs en baisse, notamment au Maghreb) et la pauvreté qui poussent les gens hors de chez eux que l'attirance pour d'autres horizons, l'absence d'espoir sur place, le désir de se réaliser, la visibilité à travers les migrants de retour le temps des vacances d'une société de consommation et de liberté d'expression. Enfin et surtout, beaucoup de nouveaux migrants, de l'Est notamment mais aussi du Sud, s'inscrivent dans une stratégie de co-présence ${ }^{3}$, ici et là-bas, surtout quand leurs titres de séjour et l'absence de visas le leur permettent : plus les frontières sont fermées, plus les gens s'installent, faute de pouvoir repartir et revenir, plus elles sont ouvertes ou entrouvertes, plus ils circulent et moins ils s'installent.

L'Europe continue à arborer une attitude frileuse face aux migrations, sous la pression de la politique de sécurisation des frontières, tandis qu'elle est confrontée au double défi du vieillissement des Européens et des pénuries sectorielles de main-d'œuvre, deux réalités mises en évidence par un rapport des Nations unies sur les migrations de remplacement de mars 2000, suivi par un Livre vert européen de 2005. Aussi, les contradictions sont légion: depuis 1974, la plupart des pays européens de l'Ouest ont suspendu l'accès de leurs frontières à l'immigration salariée, un régime d'exception comparé aux autres régions d'immigration du monde. Les effets pervers sont nombreux : entrées clandestines, sédentarisation des sans papiers, esclavage moderne, détournement de la demande d'asile et du mariage à des fins de travail. Les secteurs qui manquent de main-d'œuvre comme le bâtiment et les travaux publics, les métiers du tourisme, les services domestiques et aux personnes âgées, l'agriculture, l'habillement font appel à un volant d'immigration clandestine. La fermeture est liée au fait que les politiques européennes ont été progressivement définies autour d'une série d'idées fausses et de scénarios n'ont pas eu lieu. Il y a vingt-cinq ans, la plupart des pays européens pensaient que l'ère des grandes migrations de masse était terminée, que l'heure était à la substitution de la main-d'œuvre nationale à la main-d'œuvre étrangère et à la nouvelle division internationale du travail, aux politiques de retour et de réinsertion des non Européens dans leurs pays d'origine, à la mobilité interne des 
Européens dans une Europe sans frontières intérieures et au co-développement, comme alternative à la migration.

11 Ces prévisions ont été largement démenties par les faits et les politiques européennes, de même que celles des États, ont concentré leurs objectifs sur le contrôle des frontières, dans une vision sécuritaire habitée par le risque migratoire, le terrorisme, la criminalité organisée et l'obsession des défis à «l'intégration». Ainsi, les politiques d'immigration sont en permanent décalage avec la réalité des flux. Ce décalage est aujourd'hui accentué par les négociations en cours sur la reprise des migrations de travail (« immigration choisie » en France depuis 2006, politique des permis à points en Allemagne depuis 2005, accords bilatéraux de main-d'œuvre dans l'Europe du Sud, ouverture aux travailleurs de l'Est depuis 2006 au Royaume Uni, en Irlande et en Suède, mise en œuvre de la blue card - à l'instar de la green card américaine - à compter de novembre 2007), le souci de tisser des liens entre l'Europe et ses voisins (poursuite tolérée de l'immigration frontalière à l'Est de la Pologne et de la Roumanie, projet français d'Union méditerranéenne et de co-développement incluant les migrations) et la poursuite de la lutte contre l'immigration clandestine. Les contradictions entre ces politiques et l'absence de consensus entre les États européens sur un thème crucial pour l'identité européenne cristallisent les attentions et les crispations chez les décideurs et dans l'opinion publique, encore peu acquis à l'idée de la mondialisation du phénomène migratoire dont l'Europe est l'un des terrains d'élection.

\section{L'Europe, ses frontières et ses migrations}

12 La fermeture des frontières aux travailleurs étrangers, voici près de trente ans, a eu pour effet d'accélérer le regroupement familial (plus de $50 \%$ des entrées légales annuelles), de provoquer leur sédentarisation, et n'a pas empêché la venue d'autres flux : réfugiés, illégaux, experts, étudiants... : un phénomène où, contrairement au passé, où entraient surtout des «bras", les facteurs d'attraction (pull) sont devenus plus puissants que les facteurs qui poussent les gens hors de chez eux (push). L'Europe attire donc par elle-même. Aussi, les migrants sont-ils moins des ruraux analphabètes que du temps des migrations de masse des années 1960, mais davantage des urbains scolarisés issus des classes moyennes. Outre la migration familiale, la demande d'asile est la source qui a le plus augmenté au cours des quinze dernières années notamment en Allemagne, au Royaume-Uni et en France. L'Afghanistan, l'Irak, la Turquie, l'exYougoslavie, la Chine et l'Afrique subsaharienne y figurent en bonne place. Comparées aux principaux réservoirs démographiques d'immigration potentiels que constituent la Chine, l'Inde ou l'Irak, les migrations intra-européennes qui bénéficient pourtant de toutes les libertés d'installation, de circulation et de travail, restent faibles. Cette mobilité interne à l'Europe est la plus élevée au Luxembourg, suivi de la Suisse, de l'Irlande, du Royaume Uni, de la Belgique, du Portugal, de la Suède, de l'Espagne et de la Grèce. On constate en revanche que, depuis une vingtaine d'années, la part des étrangers originaires de pays tiers a augmenté et que certaines nationalités ont gagné en importance (migrants originaires des pays d'Europe centrale et orientale en Allemagne, Marocains et Sénégalais en France, ex-Yougoslaves aux Pays-Bas), tandis que des nationalités nouvelles s'affirment dans le paysage migratoire: Pakistanais, Vietnamiens, Iraniens, Sri Lankais, Chinois. 
Quant aux installés, dans l'Europe des 27, sur 450 millions d'habitants, on compte environ 25 millions d'étrangers, dont un quart d'Européens communautaires. Ces étrangers sont inégalement répartis dans les pays d'accueil : ainsi l'Allemagne est le premier pays d'immigration, avec 7,5 millions d'étrangers, $9 \%$ de sa population totale, suivie par la France (3,5 millions, $6 \%$ de la population totale), l'Espagne (passée d'1,5 million en 2002 à plus de 4 millions aujourd'hui) et le Royaume-Uni (2,8 millions, $4 \%$ de la population totale), la Suisse ( 1,4 million, près de $20 \%$ de la population), l'Italie (1,5 million d'étrangers, $2,4 \%$ de la population) et la Grèce (800 000 étrangers, $8 \%$ de la population totale). Mais la proportion d'étrangers n'est pas toujours liée à leur poids numérique (Luxembourg, $30 \%$ d'étrangers, Autriche $10 \%$, Finlande $2 \%$ ). Malgré la mondialisation, chaque pays a un peu « ses » étrangers, fruits de l'héritage colonial, de relations bilatérales privilégiées ou de la proximité géographique. Dans la plupart des pays européens, $60 \%$ des étrangers ne proviennent que de quatre ou cinq pays de départ, même si l'on s'achemine vers une diversification des pays d'émigration et des types de migrants et une dizaine de pays européens d'accueil seulement concentrent la presque totalité des immigrés: Allemagne, France, Espagne, Royaume Uni, Italie, Suisse, Belgique, Grèce, Autriche, Pays-Bas par ordre décroissant du nombre d'étrangers.

En Europe de l'Est, la chute du mur de Berlin a donné lieu à des migrations ethniques de retour, notamment celle des Aussiedler en Allemagne (deux millions), des Allemands installés depuis plusieurs siècles à l'Est de la frontière allemande, en Pologne, dans les pays baltes, en Russie, en Sibérie, celle des Bulgares retournés en Turquie (près d'un demi-million), des Finnois de Carélie en Finlande ${ }^{4}$, des Grecs pontiques ${ }^{5}$ en Grèce (131600), des Hongrois de Transylvanie (Roumanie) vers la Hongrie, des Italiens d'Argentine et d'ailleurs vers l'Italie (306 000), l'Autriche ayant aussi connu quelques 380000 retours ethniques. Mais les Peco (Pays d'Europe centrale et orientale) représentent surtout une migration vers l'Europe de l'Ouest, tout en accueillant une population venant d'Ukraine, de Pologne et de Roumanie. Contrairement aux idées reçues, la grande déferlante ne s'est pas produite et il s'est agi surtout de migrations de voisinage et plus encore de migrations pendulaires (Polonais en Allemagne, Roumains en Italie, Ukrainiens en Espagne et au Portugal), de la part de gens qui s'installent dans la mobilité comme mode de vie. Le groupe le plus important est celui des Polonais, suivis des Roumains et des Ukrainiens. Autre nouvelle migration : les Roms. Au nombre de 8 à 12 millions en Europe (les chiffres varient fortement d'une source à l'autre), les Roms sont surtout présents en Roumanie (deux millions), en Slovaquie, en Bulgarie et en Hongrie.

Au Sud, on trouve le même phénomène de migrations en chaîne, la Turquie étant devenue une zone de migrations et de transit pour les migrations de voisinage (soit environ 250000 étrangers, venus d'Irak, de Moldavie, d'Iran, d'Afghanistan) et le Maghreb une zone de départ et d'accueil ou de transit pour une migration subsaharienne venue du Sénégal, du Mali et de Mauritanie. La fermeture des frontières appliquée par les pays du Maghreb en application des dispositifs européens d'externalisation des frontières conduit les migrants illégaux à changer leurs parcours et à partir des côtes africaines par mer jusqu'aux îles Canaries plutôt que Gibraltar ou à traverser le désert et tenter les îles siciliennes plutôt que Brindisi, ce qui rend le voyage plus dangereux et a conduit à plusieurs milliers de morts aux abords de l'Europe depuis 2000. 
16 Partout, la mobilité est régie par des réseaux transnationaux d'origine familiale, économique, commerçante, ainsi que mafieuse qui ne sont que superficiellement affectés par les politiques de contrôle des frontières. La fascination pour l'Eldorado occidental est grande et "l'envie d'Europe» aussi, surtout pour tous ceux qui considèrent qu'il n'y a aucun espoir chez eux. L'exode rural se dirige vers de grandes métropoles de départ qui sont des espaces d'échanges où prospère une économie liée à la frontière et à sa fermeture : trafics de main-d'œuvre, d'êtres humains, de drogue, produits de contrebande mais aussi commerce tout court. De grands marchés viennent matérialiser ces zones de friction et de rencontre entre deux mondes, au départ ou à l'arrivée. Ce sont des plaques tournantes pour la migration, souvent à proximité des frontières. Parfois c'est le mariage, moins souvent blanc que traditionnel ou mixte d'ailleurs, ou les réseaux religieux (chrétiens comme musulmans) qui servent de ticket d'entrée vers le rêve européen, puisque les frontières ne sont ouvertes qu'aux plus nantis (commerçants et hommes d'affaires, experts, universitaires munis de visas à entrées multiples, titulaires de titres de séjour de longue durée ou de la nationalité d'un pays d'accueil et ainsi dispensés de visas, étudiants).

Quelques profils dominent dans ces nouveaux types de migrants : des hommes jeunes et diplômés issus des classes moyennes urbaines, nourris d'aspirations diffuses à la modernité occidentale, des femmes isolées, scolarisées, accédant à une indépendance économique et personnelle mais parfois aussi cherchant une liberté d'expression, des mineurs, souvent victimes d'exploitation en tout genre, aux motivations difficiles à démêler, des élites très qualifiées à la recherche d'une réalisation professionnelle à la mesure de leurs compétences ou de leurs talents, des hommes prêts à offrir leurs bras pour améliorer leur condition, des groupes installés dans la mobilité comme les paysans maliens de la région de Kayes, les Chinois de Wenzhou, les Roumains du pays d'Oas. Hormis les réfugiés et l'immigration matrimoniale (mariages et regroupement familial), beaucoup de ces nouveaux migrants aspirent davantage à la mobilité qu'à l'installation définitive. Ils considèrent parfois leur séjour comme un passage vers d'autres destinations plus convoitées (États-Unis, Canada) ou comme un aller-retour entre « ici » et chez eux. Tout porte à croire que ces tendances vont se poursuivre, compte tenu de la persistance des déséquilibres mondiaux, de la rencontre d'un désir d'individualisme avec un sentiment d'absence de perspectives dans les pays de départ. Cette mobilité est une source de dynamisme économique et démographique (50 \% de la population sur la rive sud de la méditerranée a moins de 25 ans), mais elle se heurte à la réticence des pays européens à prendre en compte cette nouvelle donne.

18 La Méditerranée fait un peu figure de Rio Grande, de ligne de fracture géopolitique entre l'Afrique et l'Europe. La fermeture des frontières s'y conjugue avec la libéralisation accrue des échanges commerciaux (tels étaient les objectifs du processus de Barcelone, entre 1995 et 2005) mais aussi avec l'absence d'alternative véritable à la migration. Dans le même temps, les pays d'immigration européens cherchent à limiter la part de l'immigration familiale par rapport à la migration de travail salarié, réintroduite dans plusieurs pays, en souhaitant une immigration temporaire répondant à la structure du marché du travail. Mais ils peinent à définir collectivement leurs besoins de main-d'œuvre dans le long terme et renforcent leur fermeture vis-à-vis des pays du sud, rendant ambigu le projet d'intégration régionale euroméditerranéenne. Pourtant, la dépendance des pays européens à l'égard de l'immigration a des chances 
de s'accroître, compte tenu des faibles ressources démographiques des pays européens de l'Est nouvellement entrés dans l'Union.

\section{Réponses européennes : une européanisation à reculons}

19 L'Union européenne définit le cadre des politiques de maîtrise des flux, qui passent de l'échelon intergouvernemental à la communautarisation des décisions, mais de façon désordonnée du fait des divergences et des convergences entre les politiques nationales, de la solidarité institutionnelle entre les pays signataires des accords de Schengen par rapport à ceux qui n'en sont pas signataires, de la superposition de plusieurs espaces normatifs de référence, notamment à propos du marché nordique du travail dont font partie deux pays non membres de l'Union européenne. De plus, l'harmonisation des politiques européennes de migration est porteuse de restriction de droits fondamentaux comme l'asile politique du fait de la recevabilité de la demande dans un seul pays. Elle détourne les accords de Schengen de leur finalité initiale qui était celle d'un espace de liberté de circulation, elle aggrave l'écart, quant aux droits, entre Européens et extra-Européens, substituant une nouvelle frontière juridique à celle qui opposait les nationaux des étrangers.

La dilution de la souveraineté nationale dans les processus de décision européens, la perte de pertinence de la notion de frontières et les difficultés de l'Europe à s'affirmer comme objet politique semblent compensées par une affirmation de la souveraineté de l'Union sur le contrôle de ses frontières externes. Alors que le bien fondé d'une communautarisation accrue des politiques d'immigration et d'asile dépend d'une plus grande confiance dans les instruments européens, chaque pays cherche à donner l'illusion qu'il reste maître de sa politique migratoire, vis-à-vis de son opinion publique.

\section{L'européanisation des politiques migratoires}

21 La construction de l'espace migratoire européen s'est effectuée en plusieurs étapes. Depuis la signature du traité de Rome en 1957 et la mise en place progressive de la liberté de circulation des travailleurs (1968), une étape décisive a été franchie en 1985 avec l'adoption de l'Acte Unique européen qui définit un espace européen sans frontière grâce à la liberté de circulation des personnes, et non plus seulement des travailleurs, et la signature des accords de Schengen (1985) qui avaient pour objet de réaliser le laboratoire pour l'Acte Unique. Ses principaux instruments sont :

- l'adoption d'un visa unique de moins de trois mois, obligatoire pour les non communautaires qui veulent pénétrer et circuler en touristes dans l'espace Schengen ;

- la liberté de circulation à l'intérieur des frontières européennes pour les Européens et les détenteurs (non communautaires) d'un visa Schengen et le renforcement des frontières extérieures de l'Union grâce à l'adhésion progressive au système Schengen des nouveaux entrants et à la solidarité entre les pays européens dans les contrôles externes menés par les pays situés sur les frontières extérieures de l'Europe. Des accords de réadmission sont signés à partir de 1991 avec les pays non communautaires riverains ou voisins de l'Union européenne en vertu desquels les États s'engagent à 
reprendre sur leur territoire les clandestins dont on a pu établir qu'ils étaient leurs ressortissants ou qu'ils avaient transité par chez eux ;

- l'adoption d'un système informatisé de contrôle, le SIS (Système d'information Schengen) pour la mise en ligne des données nationales sur les "indésirables » (clandestins, déboutés du droit d'asile), obligeant tous les États européens à leur refuser le droit au séjour et à les expulser ; - une politique d'asile commune, définie en 1990 par les accords de Dublin à l'échelon de l'Europe des Quinze, assortie d'un dispositif de filtrage renforcé : notion de pays sûr, d'où on ne peut pas demander l'asile, de demande manifestement infondée, de sanctions contre les transporteurs, solidarité entre pays européens dans le contrôle à l'entrée, un demandeur d'asile débouté ne pouvant, sauf exception, demander l'asile dans un autre pays de l'Union (pour éviter les demandes d'asile multiples et «en orbite »). Le protocole Aznar spécifie qu'il n'est désormais plus possible de demander l'asile en Europe quand on est originaire d'un pays de l'Union européenne (1997). Les accords de Dublin II de 2003 ajoutent que les demandeurs d'asile doivent être examinés dans le premier pays de l'Union où ils mettent le pied (" one stop, one shop »).

- la lutte contre l'immigration clandestine. Des règles communes sont définies à l'échelon européen pour lutter contre le séjour illégal (1990), harmoniser le regroupement familial et définir une "préférence européenne " à l'emploi (1994) qui protège l'emploi des nationaux et des Européens des nouveaux entrants non communautaires sur le marché du travail. En décembre 2000 une convention (Eurodac) sur l'asile a été établie pour le contrôle des empreintes digitales des demandeurs d'asile et des personnes ayant franchi irrégulièrement une frontière à partir d'une base de données informatique. L'accès à l'information est ouvert à chaque État membre de l'Union européenne. Le contrôle renforcé des frontières est aussi symbolisé par le Sive (système intégré de vigilance externe) à l'aide de radars entre l'Espagne et les côtes africaines. À Séville, en juin 2002, les États européens ont décidé d'accélérer le processus d'harmonisation des politiques migratoires dans le sens de plus d'« équilibre", mais ils se sont surtout focalisés sur la lutte contre l'immigration clandestine et l'abus des demandes d'asile: clauses de réadmission, gestion conjointe des flux migratoires (opération "Ulysse » coordonnée par l'Espagne visant à lutter contre l'immigration illégale en mer). Cette tendance sécuritaire a été confirmée par les sommets de Thessalonique (2003) et de La Haye (2004). Des accords de réadmission entre l'Union européenne tendent à faire de nombreux États tampons les «gardefrontières » de l'espace européen, d'autres États (africains notamment) étant déjà liés par une clause de réadmission obligatoire. Des officiers de liaison immigration et asile à travers le programme Frontex, formalisé dans une agence spécialisée, assurent un contrôle renforcé des frontières externes. Le rapatriement communautaire (c'est-àdire par plusieurs pays de l'Union, qui joignent leurs efforts de façon conjointe) est considéré comme un signal fort de dissuasion.

27 - la citoyenneté européenne, définie en 1992 par le traité de Maastricht qui fait de la liberté de circulation, d'installation et de travail l'un des attributs essentiels de celle-ci, en son article 8, avec le droit de vote et l'éligibilité locale et au Parlement européen pour les Européens résidant dans un autre pays que celui dont ils ont la nationalité.

28 - le passage du « troisième pilier intergouvernemental de l'immigration et de l'asile ${ }^{6}$ au premier pilier communautaire avec, en 1997, le traité d'Amsterdam qui intègre «l'acquis Schengen » dans le traité de l'Union européenne. Mis en œuvre à partir de 
1999 pendant une période transitoire de cinq ans, ce dispositif introduit un changement du processus de décision, passant de l'unanimité à la majorité qualifiée. Ce dispositif devrait être mis en œuvre de façon conjointe entre la Commission (à la majorité qualifiée) et le Parlement européen à partir de la mise en œuvre du traité de Lisbonne de 2007. Les législations nationales relatives à l'entrée et à l'asile s'harmonisent peu à peu mais la communautarisation est empreinte d'une idéologie sécuritaire et restrictive.

29

- la définition, avec, en 1999, le sommet de Tampere d'une politique d'immigration commune à partir d'une évaluation des besoins économiques et démographiques de l'Union européenne et de la situation des pays d'origine, et l'abandon de l'objectif de "l'immigration zéro ». À Laeken (décembre 2001) les pays européens ont évoqué un " équilibre nécessaire » entre la protection des réfugiés, l'aspiration légitime à une vie meilleure et la «capacité d'accueil des États membres. Cette évolution est interprétée comme une reprise de l'immigration de main-d'œuvre légale malgré l'ambiguïté maintenue sur la question des quotas: en 2007 le commissaire européen à l'immigration, M. Frattini, a annoncé le lancement de la blue card ${ }^{7}$, symbole de la reprise de l'immigration de travail vers l'Europe en fonction des besoins européens. Mais beaucoup d'États membres continuent à "faire leur marché " de main-d'œuvre, qualifiée ou non, sur la scène internationale, sous la forme d'accords bilatéraux de voisinage, de sous-traitance et de travail au noir plus ou moins toléré selon les secteurs. Pour les très qualifiés, la "stratégie de Lisbonne », lancée au Conseil européen de 2000 cherche à favoriser en Europe l'économie de la connaissance la plus compétitive du monde d'ici 2010.

La complexité du système européen renforce le registre du contrôle (coopération européenne renforcée, adoption de l'acquis communautaire par les États entrés dans l'Union), tout en faisant progresser lentement l'harmonisation des politiques européennes. La superposition de plusieurs espaces normatifs de référence et de soussystèmes partiellement intégrés à l'Union tend à créer une "Europe à la carte ». Le Royaume-Uni, l'Irlande du Nord et la république d'Irlande ne participent pas à la politique commune d'immigration et d'asile, bien qu'ils aient signé la Convention de Dublin sur l'examen des demandes d'asile. Quant au Danemark, membre de Schengen, il n'a pas souhaité être partie prenante des délibérations du Titre IV du traité d'Amsterdam sur l'immigration et l'asile, bien qu'il participe à la politique commune des visas. L'Islande et la Norvège, qui appartiennent à l'Union douanière nordique et les nouveaux pays entrés dans l'Union européenne sont en revanche tenus d'adopter l'intégralité de l'acquis Schengen et de la coopération en matière de Justice et d'Affaires intérieures. Les politiques européennes qui ont ouvert les frontières de l'Union à la libre circulation des Européens de l'Est (y compris de l'Allemagne d'aujourd'hui) dès 1991 (pays dits de Visegrad exemptés de visas de court séjour: Pologne, Hongrie, république tchèque, puis Slovénie et Estonie, les derniers en date étant la Bulgarie au 31 décembre 2000 et la Roumanie au 31 décembre 2001) leur accordent la liberté d'installation et de travail en ordre dispersé, selon les pays d'accueil et les pays de départ. Les accords sur le libre accès au marché du travail des Européens de l'Est prévoient un temps d'attente d'ici 2009 mais certaines nationalités peuvent déjà travailler légalement dans certains pays (Suède, Irlande et Royaume Uni depuis 2004 où les Polonais sont venus nombreux). 
31 Les événements du 11 septembre 2001, s'ils n'ont pas eu d'impact majeur sur les politiques migratoires européennes, ont néanmoins renforcé le volet sécuritaire et associé les représentations de l'immigration à la criminalité et au terrorisme islamiste : une tendance qui existe de longue date, depuis que les Ministères de l'Intérieur des États-membres ont tenté au tournant des années 1990 de s'approprier la "gouvernance» des questions migratoires. Pourtant les défis sont tout autres et parfois contradictoires: démographie, intégration, pénuries de main-d'œuvre, maintien de l'État-providence, libéralisme économique, respect des droits de l'homme.

\section{Politiques des États}

32 La marche à l'européanisation est entravée par la forte dépendance de chacun des États à l'égard de son opinion publique, de son marché du travail, de sa démographie, de son voisinage avec des pays faisant ou non partie de l'Union européenne, de sa diplomatie, de la diversité de ses flux migratoires du fait de l'histoire (ancienneté ou non de la migration, passé colonial ou non), de la géographie (insularité ou frontières terrestres), et des réseaux familiaux, économiques, culturels, linguistiques existants avec telle ou telle région du monde.

33 La plupart des pays européens ont répondu à la pression migratoire par la modification fréquente des lois en vigueur sur l'entrée, le séjour et la nationalité ainsi que par des vagues de régularisations successives : durcissement des politiques d'entrée et du droit d'asile, externalisation des frontières à distance, maintien de l'opposabilité de l'emploi pour les non Européens qui cherchent à entrer sur le marché du travail lors de leur arrivée, restrictions temporaires apportées à l'accès au marché du travail des nouveaux Européens de l'Est.

Malgré la faveur actuelle de la politique d'immigration choisie (y compris dans les nouvelles politiques d'immigration des pays d'Europe de l'Est), la migration de masse est considérée comme une exception historique, avec vocation éventuelle au retour, malgré la sédentarisation du plus grand nombre. Les politiques d'entrée s'inspirent de cette réticence à reconnaître la légitimité de la présence étrangère : pressions de l'opinion publique, introduction de tests linguistiques et d'apprentissage de valeurs civiques dans les politiques d'entrée, manifestations de xénophobie, crainte d'une invasion venue du Sud et de l'Islam.

35 Si les procédures sont harmonisées à l'entrée ou en passe de l'être, les dispositions relatives au séjour restent du ressort de la souveraineté des États (en fonction de la règle de la subsidiarité). Elles sont à la source de grandes disparités entre les pays d'accueil: durée des titres de séjour variable, admission au travail immédiatement après l'entrée ou à la suite d'un temps de présence défini, accès variable aux droits sociaux, diversité de l'interprétation de la Convention de Genève relative à l'asile, du traitement social des demandeurs d'asile en cours de procédure - avec ou non droit au travail -, pluralité des modalités du regroupement familial (quant aux ayants droit) et des codes de la nationalité donnant accès à l'acquisition de celle du pays d'accueil. Tous ces éléments viennent s'ajouter à l'attraction diverse et sélective du marché du travail, des salaires, des prestations sociales, des niches d'emploi sectorielles ou de l'installation, préalable ou non, de réseaux familiaux ou relationnels sur place dans chaque pays d'accueil. 

Espagne, Portugal et Grèce) qui ont régularisé le plus les clandestins. Certains pays du nord y ont vu un facteur d'appel, sans que la démonstration en ait été faite. Ainsi, l'Italie (en 1986, 1990, 1995, 1998, 2003, 2005), y a procédé abondamment, suivie de l'Espagne (en 1991, 1996, 2000, 2006), du Portugal (en 1993, 1996) et de la Grèce. Mais d'autres pays européens y ont eu recours aussi : la France (en 1981 et 1997) et la Belgique (en 2000), y compris en Allemagne aujourd'hui.

Il en va de même pour l'asile. Les pays européens sont, du fait de leur passé colonial pour certains, de leur situation géographique pour d'autres, de leur tradition en matière d'asile pour d'autres encore, diversement confrontés au phénomène des réfugiés. S'y ajoutent de sensibles différences juridiques sur l'interprétation du droit d'asile et sur les critères donnant droit au statut, ce qui rend difficile le traitement de la question à l'échelle communautaire. Ainsi, depuis 1945, l'Allemagne de l'Ouest a accueilli à elle seule près de la moitié de l'ensemble de demandeurs d'asile qui frappaient aux portes de l'Europe occidentale du fait de son droit d'asile constitutionnel (article 16.2 de la Loi Fondamentale du 23 mai 1949, modifié par la loi de 1993). Les pays européens les plus concernés (Allemagne, Autriche), sont favorables à un « partage du fardeau ».

Les réponses des pays d'accueil aux nouveaux profils de demandeurs d'asile, qui fuient à la fois la pauvreté, la crise politique ou la guerre civile sont variables car ils rechignent souvent à reconnaître la situation des pays de départ comme définitive, de peur de cautionner ainsi la victoire contestée d'un groupe ou d'une ethnie. D'où l'apparition de réfugiés humanitaires, au séjour temporaire, le développement de l'asile externe au gré des États d'accueil, l'assignation dans des camps, la délivrance de visas de transit vers d'autres destinations, la création de zones internationales dans les aéroports : autant de dispositifs aussi provisoires que discrétionnaires, dépendants de politiques publiques nationales, alors que la politique d'asile se veut européenne. Les grandes tendances à la baisse du taux de reconnaissance du statut de réfugié s'inscrivent aussi dans l'absence d'harmonisation des critères à l'échelon européen et la difficulté d'homogénéiser des procédures souvent juridictionnelles, donc indépendantes.

Enfin, d'autres disparités proviennent des situations migratoires dans les pays d'accueil. Disparité quant au volume et à la concentration des nationalités. Si les Turcs (plus de trois millions) sont les plus nombreux, suivis des Marocains, certaines nationalités ne vivent que dans un seul pays ( $97 \%$ des Algériens, les deux tiers des Portugais et des Tunisiens et $50 \%$ des Marocains immigrés en Europe vivent en France), $80 \%$ des Grecs, $72 \%$ des Turcs, $68 \%$ des Polonais et des ex-Yougoslaves vivent en Allemagne ; la presque totalité des Irlandais et des ressortissants du Commonwealth se trouvent au Royaume-Uni. On trouve alors des implantations de type quasidiasporique, entretenant des réseaux entre elles et les pays d'origine et d'autres qui se trouvent dans une relation de couple migratoire avec le pays d'accueil, situation souvent héritée d'un passé colonial (Indo-Pakistanais au Royaume-Uni, Maghrébins en France, Turcs en Allemagne). Mais les pays européens doivent aussi faire face à une immigration plus mobile qui n'aspire plus nécessairement à la sédentarisation. De plus, malgré la marche vers une certaine convergence des droits des résidents, et du droit de la nationalité vers un équilibre entre le droit du sol et le droit du sang, d'autres frontières intérieures se construisent autour de la ségrégation urbaine et des 
discriminations où les politiques locales ont un rôle essentiel. Elles relèvent du principe de subsidiarité, inspiré du système fédéral allemand, en vertu duquel l'intervention de l'Union européenne se limite aux domaines pour lesquels l'action engagée ne peut être réalisée de manière suffisante par les États membres.

Aujourd'hui les pays européens semblent hésiter entre le modèle de l'immigration d'installation et celui des travailleurs hôtes.

\section{Perspectives et prospective : co-développement, quotas, droit à la mobilité}

41 Depuis 2000, un débat nouveau est venu bousculer le consensus sécuritaire et le credo de l'«immigration zéro»: le vieillissement à l'horizon 2030 de la population européenne, les pénuries sectorielles de main-d'œuvre et les déséquilibres entre la part des actifs et des inactifs nécessitent une autre approche des migrations, fondées sur la libre circulation, la sécurisation des parcours et le multilatéralisme de la décision dans la gouvernance des migrations. Ce constat est difficilement accepté par les États européens, habitués à la fermeture. Mais l'entr'ouverture est néanmoins entrée dans l'agenda de la politique européenne des migrations. Cette nouvelle inflexion s'inspire d'une seconde priorité affichée en faveur de l'ouverture : la compétition mondiale pour l'attraction des plus qualifiés, la croissance de la population en Europe mais aussi l'inscription de la mobilité d'une population jeune parmi les «biens publics mondiaux ». Un autre objet de controverse réside dans les relations que la migration entretient avec le développement dans les pays de départ.

À court terme (c'est-à-dire celui des politiques migratoires), le temps des migrations ne coïncide pas avec les stratégies de développement : plus il y a de développement, plus il $\mathrm{y}$ a de migration et plus il y a de migration, plus il y a de développement du fait des transferts de fonds et du mieux être apporté par l'immigration même s'il ne s'agit pas de développement durable créateur d'emplois. Pour le candidat au départ, nourri d'imaginaire, l'anticipation d'un éventuel développement de sa région à long terme passe souvent après la tentation migratoire, même périlleuse ou mortelle. Il en va de même des élites qui ne s'estiment pas toujours devoir servir un pays qui ne leur offre rien. Sous l'effet de la mondialisation, les pauvres ont connaissance de la richesse du Nord et savent que si elle ne vient pas à eux, c'est eux qui s'efforceront d'aller à elle grâce aux réseaux qui les mettent en mouvement. La question de l'inclusion est un autre défi pour les pays européens.

43 Face au décalage entre les mécanismes européens de maîtrise des flux migratoires et les réalités qui se dessinent, la communautarisation des décisions européennes est un instrument plus performant que les politiques étatiques pour y répondre mais elle reste empreinte de bien des confusions et des hypocrisies. On assiste aujourd'hui à une reprise des migrations économiques. Pour l'Europe l'enjeu consiste à trouver un compromis entre la fermeture et l'ouverture, entre la logique sécuritaire et celle des marchés. Dans le même temps, le droit à la mobilité, la démocratisation des frontières font partie des droits émergents et se profilent des tentatives de gouvernance mondiale des migrations (Conférences de New York de septembre 2006 et de Bruxelles de juillet 2007) associant pays de départ, d'accueil, OIG et ONG, associations de migrants, pour que la porte de service ne soit plus systématiquement utilisée à défaut de la porte principale. La France a inscrit l'immigration parmi les trois priorités de sa présidence 
de l'Union à partir de juillet 2008. Peut-on espérer que le réalisme présidera enfin à la dépendance électorale des politiques migratoires?

\section{BIBLIOGRAPHIE}

Bade K., 2002, L'Europe en mouvement, Paris, Seuil.

Bertossi C., 2007, «L'Europe en mal de migrations ? ", in Montbrial T. (de), Moreau Defarges Ph. (dir.), L'Europe et le monde, RAMSES, Paris, Dunod, p. 81-91.

Bribosia E., Rea A. (dir.), 2002, Les nouvelles migrations. Un enjeu européen, Bruxelles, Complexe.

Castles S., 2006, " Guestworkers in Europe : A Resurrection ? ", International Migration Review, 40 (4), p. 741-766.

OCDE, 2007, Tendances des migrations internationales, SOPEMI, Paris.

Tinguy A. (de), 2004, La grande migration, Paris, Plon.

Wihtol de Wenden, C., 2005, Atlas des migrations dans le monde, Paris, Autrement, 2005.

Wihtol de Wenden, C., 2007, « L'Union européenne et les enjeux migratoires », in Chopin T., Foucher M. (dir.), L'État de l'Union 2007, Paris, Fondation Robert Schuman, p. 111-117.

\section{NOTES}

1. On appelle "migrations en chaîne » la spirale des migrations induite par la mobilité : le départ des uns entraîne des besoins de main-d'œuvre donc de nouveaux migrants dans les zones de départ, tout en construisant une chaîne migratoire vers les pays d'accueil.

2. Ce terme a surtout été utilisé pour analyser les migrations est-ouest en Europe lors de la chute du mur de Berlin.

3. Selon le terme de Serge Weber, Des chemins qui mènent à Rome, Thèse de géographie, Université Paris I, décembre 2004. Cela s'oppose à la «double absence » des migrants des années 1960 et 1970 décrits par Abdelmalek Sayad et signifie l'existence d'une double vie entretenue par des liens permanents économiques, familiaux et affectifs.

4. La délimitation de la frontière par Molotov entre la Russie et la Finlande après la seconde guerre mondiale selon un découpage non rectiligne (le « doigt de Molotov » dépassant de la règle qui a servi à la délimiter) a mis du côté soviétique une partie des Finlandais qui sont pour partie retournés ensuite en Finlande depuis 1990.

5. Il s'agit de colonies grecques installées de longue date dans la région dite du Pont Euxin, c'està-dire à l'est de la mer Noire, en Turquie.

6. On entend par " pilier » un champ de compétence communautaire correspondant à une étape de la communautarisation des décisions, le premier étant l'économie, le second la politique étrangère et le troisième la justice et les affaires intérieures.

7. Il s'agit d'une carte de séjour portant autorisation de travail salarié dans les secteurs qualifiés dont l'Europe a besoin et pour lesquels elle lève la préférence européenne à l'emploi, un système institué en 1994 pour protéger le travail des Européens de l’Union. 


\section{RÉSUMÉS}

L'Europe est confrontée à un paradoxe: d'un côté, elle est devenue l'une des premières destinations migratoires du monde depuis ces vingt dernières années, dans un contexte de manque de main-d'œuvre sectorielle et de vieillissement. De l'autre, elle continue à inscrire les migrations dans le registre sécuritaire de la dissuasion et de la lutte contre l'immigration clandestine comme objectif prioritaire. Les réponses politiques à ces éléments contradictoires se traduisent par le décalage ("migration gap»), que l'on constate aussi aux États-Unis : décalage entre les impératifs étatiques devenus européens de maîtrise des frontières et les lois du marché, décalage entre l'insertion dans une compétition mondiale pour les compétences et talents et la frilosité des politiques d'accueil, décalage entre l'affichage des droits de l'homme et les difficultés $\mathrm{du}$ « containment ». L'européanisation des politiques migratoires de gestion des flux s'accompagne de surcroît d'une très grande diversité des situations et des réponses politiques des pays d'accueil qui se réfugient souvent dans l'«opting out», un attentisme légitimé par la spécificité des situations de chacun. Il s'y ajoute l'inscription des politiques d'intégration dans le régime de la subsidiarité, qui privilégie leur traitement à l'échelon national et local plutôt qu'européen. Cette analyse débouche sur quelques interrogations : le dialogue avec la rive sud de la Méditerranée, la politique de voisinage à l'est et l'ambiguïté des relations entre migrations et développement pour l'Europe.

Europe is facing with a paradox : on one side, it has become one of the first regions of migrations in the world during these last twenty years, in a context of shortages of labour force and of older populations. On the other side, it goes on to include migration inside the security issue, considering the fight against illegal migration as a priority. The political responses to this contradiction lead to a migration gap, yet analyzed in the United States : the gap between State policies of border control, which have become European, and the market laws, the gap between the entrance into a world competition for elites and qualified people and the reluctance of welcome policies, the gap between the assertion of Human rights and the difficulties of containment. Moreover, the europeanisation of migration policies is coupled with a great diversity of situations and political responses of welcome countries which often prefer the policy of opting out before implementing European measures. The subsidiarity which rules integration policies, managed at local or national level adds to the mosaic. This analysis opens the floor to some questions : the future of the Mediterranean dialogue, of the neighbourhood policy at East, and the ambiguity of the relations between migrations and development for Europe.

\section{INDEX}

Mots-clés : Union européenne, flux migratoires, immigration clandestine, frontières, intégration européenne

Index géographique : Europe

\section{AUTEUR}

\section{CATHERINE WIHTOL DE WENDEN}

Directrice de recherche, CERI 